\title{
Optimal solutions for fixed head short-term hydrothermal system scheduling problem
}

\author{
Thanh Long Duong ${ }^{1}$, Van-Duc Phan ${ }^{2}$, Thuan Thanh Nguyen ${ }^{3}$, and Thang Trung Nguyen ${ }^{4 *}$ \\ ${ }^{1,3}$ Faculty of Electrical Engineering Technology, Industrial University of Ho Chi Minh City, Ho Chi Minh City 700000, \\ Vietnam \\ ${ }^{2}$ Faculty of Automobile Technology, Van Lang University, Ho Chi Minh City 700000, Vietnam \\ ${ }^{4}$ Power System Optimization Research Group, Faculty of Electrical and Electronics Engineering, Ton Duc Thang University, \\ Ho Chi Minh City 700000, Vietnam
}

\begin{tabular}{l}
\hline Article Info \\
\hline Article history: \\
Received Mar 6, 2020 \\
Revised Dec 11, 2020 \\
Accepted Dec 30, 2020 \\
\hline
\end{tabular}

\section{Keywords:}

Hydrothermal system Electricity generation cost Particle swarm optimization Hydraulic constraint Power system.

\begin{abstract}
In this paper, optimal short-term hydrothermal operation (STHTO) problem is determined by a proposed high-performance particle swarm optimization (HPPSO). Control variables of the problem are regarded as an optimal solution including reservoir volumes of hydropower plants (HdPs) and power generation of thermal power plants (ThPs) with respect to scheduled time periods. This problem focuses on reduction of electric power generation cost (EPGC) of ThPs and exact satisfactory of all constraints of HdPs, ThPs and power system. The proposed method is compared to earlier methods and other implemented methods such as particle swarm optimization (PSO), constriction factor (CF) and inertia weight factor (IWF)-based PSO (FCIW-PSO), two time-varying acceleration coefficient (TTVACs)-based PSO (TVAC-PSO), salp swarm algorithm (SSA), and Harris hawk algorithm (HHA). By comparing EPGC from 100 trial runs, speed of search and simulation time, the suggested HPPSO method sees it is more robust than other ones. Thus, HPPSO is recommended for applying to the considered and other problems in power systems.
\end{abstract}

Copyright $(0) 2020$ Institute of Advanced Engineering and Science. All rights reserved.

\section{Corresponding Author:}

Thang Trung Nguyen,

Power System Optimization Research Group,

Faculty of Electrical and Electronics Engineering,

Ton Duc Thang University,

19 Nguyen Huu Tho street, Tan Phong ward, District 7, Ho Chi Minh City, Vietnam.

Email: nguyentrungthang@tdtu.edu.vn

\begin{tabular}{|c|c|}
\hline \multicolumn{2}{|l|}{ Nomenclature } \\
\hline$V o l_{h, i}$ & Volume of the $h t h \mathrm{HdP}$ in the ith period \\
\hline $\operatorname{Inf} f_{h, i}$ & Inflow of the $h t h \mathrm{HdP}$ in the ith period \\
\hline $\operatorname{Dis}_{h, i}$ & Discharge of the $h t h \mathrm{HdP}$ in the ith period \\
\hline $\operatorname{Vol}_{h, \min }, \operatorname{Vol}_{h, \max }$ & Minimum and maximum volume of the $h t h \mathrm{HdP}$ \\
\hline$D i s_{h, \min }, D i s_{h, \max }$ & Minimum and maximum discharge of the $h t h \mathrm{HdP}$ \\
\hline$x_{h}, y_{h}, z_{h}$ & Given coefficients in generation function of the hth HdP \\
\hline$P_{h, \min }, P_{h, \max }$ & Minimum and maximum power generation of the $h t h \mathrm{HdP}$ \\
\hline$P_{h, i}$ & Generation of the $h t h \mathrm{HdP}$ in the ith period \\
\hline
\end{tabular}




$$
\begin{array}{ll}
P_{t, \min }, P_{t, \max } & \text { Minimum and maximum power generation of the } t t h \mathrm{ThP} \\
P_{\text {Load }, i}, P_{\text {Loss }, i} & \text { Power of load and loss in all branches } \\
N_{p} & \text { Population size }
\end{array}
$$

\section{INTRODUCTION}

Short-term hydrothermal operation (STHTO) problem considers optimal power generation of hydropower plants (HdPs) and thermal power plants (ThPs) with intent to reduce amount of fossil fuel with very high electric power generation cost (EPGC). In general, this problem takes into account optimization time period from one operation day to one operation week [1]. The problem was considered to be complicated since it considered a hydraulic-constraint set from HdPs such as discharge boundaries through turbines, water levels of each HdP in each period and generators' boundaries. Basically, STHTO problem is divided into variable head (VH) and fixed head (FH) models in which water head is not a constant during the optimization periods in VH model but the it is fixed in FH model [2]. In recent years, ThPs and HdPs have been studied for reaching better electricity power quality by using automatic generation control [3-6]. In addition, since renewable energies were developed and installed in power systems as a main power source like ThPs and HdPs, the concern of improving electricity power quality [7]. Different areas in the same power system are supplied by either ThPs or HdPs, and both ThPs and HdPs together with renewable energies. These studies indicate that the power generation combination of ThPs and HdPs is a very important issue in power system. Thus, in this paper, the power generation combination for ThPs and HdPs continue to be optimized by selecting FH model as the main characteristic of the combined system.

The STHTO problem has been solved successfully so far by using classical approaches (CAs) and metaheuristic algorithms (MHAs). CAs [2, 8] are mainly based on taking partial derivatives of Lagrange optimization function with respect to discharge and power generation of ThPs whereas MHAs [9-19] could deal with the problem more easily. CAs are Gradient search-based method (GSA) [2], Newton-Raphson method (NRM) [8] and Lagrange function-based method [2]. The three approaches have the same characteristic in finding the optimal parameters, which is to take partial derivatives and must approximate function as linear functions. So, as valve effects of ThPs are taken into account, these methods are unsuccessful in taking the partial derivatives. Furthermore, as taking more constraints into account, more control parameters must be used in Lagrange function, leading to more difficulties in taking partial derivatives. Another disadvantage from the CAs is that they must be influenced by initial points from the starting search process. Different initial points can result in different achieved results but the same initial points always obtain the same outputs. For enhancing the robustness of CAs, estimation approaches should be used to allocate the most appropriate initial inputs. Derived from the drawbacks, the application range of CAs has not been widen in recent years. On the contrary, MHAs are much stronger in dealing with constraints and taking nonlinear or non-differentiable functions. A lot of MHAs have been applied such as simulated annealing approach (SAA) [9], evolutionary programming approach (EPA) [10-11], modified EPA (MEPA) [12], Fast EPA (FEPA)[12], improved version of FEPA (IFEPA) [12], running IFEPA (RIFEPA) [13], Clonal selection optimization approach (CSOA) [14], cuckoo search approach (CSA) [15], Gaussian distribution-based CSA (GCSA) [15], Cauchy distribution-based CSA (CCSA) [15] and Levy distribution-CSA (LCSA) [15], one rankbased CCSA (CORCSA) [16], one rank-based LCSA (LORCSA) [16], adaptive CSA (ACSA) [17], improved CSA (ICSA) [18], modified CSA (MCSA) [19], and adaptive and selective CSA (ASCSA) [19]. In general, all MHAs can solve the problem successfully and effectively; however, the complex of employed systems has not been considered as a good evidence in approximately all these methods, excluding applications of CSA variants [16-19]. A main system with the presence of one ThP and one HdP operated in a three-day plan with six periods was used to test these methods. Furthermore, only the quadratic function was used for the case of neglecting valve effects of ThPs. On the contrary, large scale systems with more complicated objective function were developed in studies [19]. Approximately all these methods have not shown persuasive evidences to demonstrate real performance of methods because only minimum EPGC has been compared.

In this paper, operation parameters of one STHTO system including ThPs' power generation and volumes of HdPs are determined for getting the minimum EPGC of all ThPs. The system is solved by implementing PSO [20], FCIW-PSO [21-22], TVAC-PSO [23], SSA [24], HHA [25] and a proposed high-performance PSO (HPPSO). Among the six employed methods, HPPSO is the modified version of PSO by using CF, IWF and TTVACs. The proposed HPPSO can be more effective than other PSO versions because it can take the advantages of CF, IWF and TTVACs. In the velocity update process of FCIW-PSO, IWF is multiplied by the old velocity and then the result and two other increased terms are added. The obtained sum is not accepted as the new velocity but it and CF are multiplied together to reach the new velocity. The two acceleration coefficients in FCIW-PSO are constant and 
normally set to 2.05. But in TVAC-PSO, they are changed within a starting value to a end value with respect to the change of present iteration. The two factors are used to adjust the second term and the third term of the new velocity while IWF is used to control the first term of new velocity. IWF is also changed from small values to high values meanwhile CF is in charge of narrowing the limit of the new velocity. As a result, the proposed HPPSO can have all strong points from other PSO versions and it is really effective for the studied problem in the paper. In the summary, the contributions of the paper are as follows:

1) Show main shortcomings of conventional PSO

2) Apply recent metaheuristic algorithms including SSA and HHA

3) Propose a new PSO method, which is effective for Optimal short-term hydrothermal scheduling problem

4) Clarify the outstanding performance of the proposed PSO over other existing PSO methods

\section{FORMULATION OF STHTO PROBLEM}

In the section, the STHTO problem is mathematically expressed by using objective and constraints. It is supposed that a typical hydrothermal system with ThPs and HdPs scheduled in optimization periods are producing and supplying electricity to loads via a load bus. A typical hydropower system is depicted in Figure 1. The objective function and all constraints can be mathematically formulated as follows:

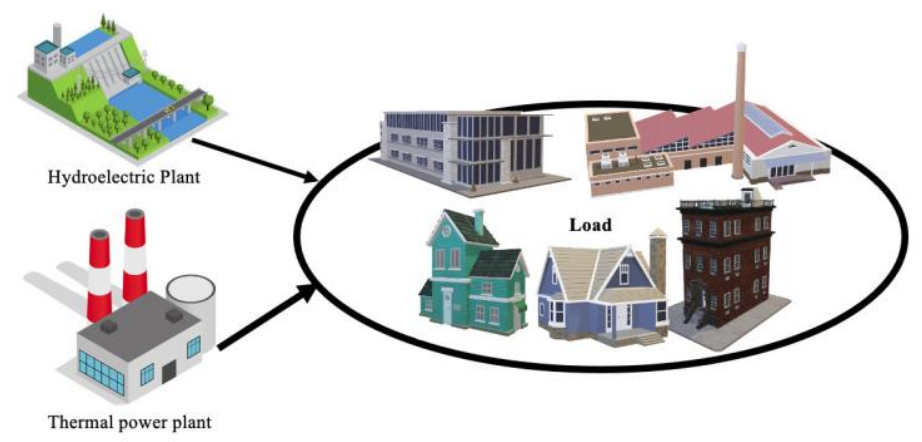

Figure 1. A typical hydrothermal power system

\subsection{Objective function}

The main target of the problem is to minimize EPGC of all ThPs. The EPGC of each thermal power plant (ThP) is a function of power generation and coefficients [21]. For the case of considering valve effects, the EPGC function is as follows:

$$
E P G C=\sum_{i=1}^{N 2} \sum_{t=1}^{N_{1}} T_{i}\left(k_{t}+m_{t} P_{t, i}+n_{t}\left(P_{t, i}\right)^{2}+\left|l_{t} \times \sin \left(s_{t} \times\left(P_{t, \min }-P_{t, i}\right)\right)\right|\right)
$$

Where

$k_{t}, m_{t}, n_{t}, l_{t}, s_{t}$ are given coefficients in EPGC function of the $t$ th ThP,

$P_{t, i}$ is power generation of the $t$ th $\mathrm{ThP}$,

$T_{i}$ is duration of the $i$ th time period,

$N_{l}$ and $\mathrm{N} 2$ are the number of ThPs and time periods.

\subsection{The set of constraints}

\subsubsection{Constraints from hydroelectricity plants:}

Water balance in reservoirs: Volume of reservoir, inflows and discharge at each period must satisfy the model below:

$$
\operatorname{Vol}_{h, i-1}-\operatorname{Vol}_{h, i}+\operatorname{Inf}_{h, i}-\operatorname{Dis}_{h, i}=0 ; h=1, \ldots, N_{3} \& i=1, \ldots, N_{2}
$$

Where $N_{3}$ is the number of HdPs.

For the cases that $i=1$ and $i=N_{2}$, volume of reservoir is constrained by:

$$
\begin{aligned}
& \operatorname{Vol}_{h, i-1}=\operatorname{Vol}_{h, \text { avai }} ; i=1 \\
& \operatorname{Vol}_{h, i}=\operatorname{Vol}_{h, \text { end }} ; i=N_{2}
\end{aligned}
$$

IJEEI, Vol.8, No. 4, December 2020: 648 - 657 
Where $\mathrm{Vol}_{h, \text { avai }}$ and $\mathrm{Vol}_{h, \text { end }}$ are available water volume before the first period and remained volume after the $\mathrm{N}_{2} t h$ period. Boundaries of volume and discharge: Volume of reservoir and discharge through turbines are limited within power bound and upper bound as follows:

$$
\begin{aligned}
& \left\{\begin{array}{l}
\operatorname{Vol}_{h, i} \geq \operatorname{Vol}_{h, \text { min }} \\
\operatorname{Vol}_{h, i} \leq \operatorname{Vol}_{h, \text { max }}
\end{array} ; h=1, \ldots, N_{3} ; i=1,2, \ldots, N_{2}\right. \\
& \left\{\begin{array}{l}
\operatorname{Dis}_{h, i} \geq D i s_{h, \text { min }} \\
\operatorname{Dis}_{h, i} \leq D i s_{h, \text { max }}
\end{array} ; h=1, \ldots, N_{3} ; i=1, \ldots, N_{2}\right.
\end{aligned}
$$

Where Dish,i is a function of hydro generation as follows:

$$
\operatorname{Dis}_{h, i}=x_{h}+y_{h} P_{h, i}+z_{h}\left(P_{h, i}\right)^{2}
$$

Boundaries of hydro generation: Hydro generation is constrained by:

$$
\left\{\begin{array}{l}
P_{h, i} \geq P_{h, \min } \\
P_{h, i} \leq P_{h, \max }
\end{array} ; h=1, \ldots, N_{3} ; i=1, \ldots, N_{2}\right.
$$

\subsubsection{Constraint of thermal generation}

ThPs are not constrained by fuel but capacity at each time period is constrained within a range as follows [27].

$$
\left\{\begin{array}{l}
P_{t, i} \geq P_{t, \text { min }} \\
P_{t, i} \leq P_{t, \text { max }}
\end{array} ; t=1, \ldots, N_{1} ; i=1, \ldots, N_{2}\right.
$$

\subsubsection{Constraints of power system}

Real power balance is a serious constraint in power system due to the stability of frequency [28]. So, the constraint below must be exactly met.

$$
\left(\sum_{t=1}^{N_{1}} P_{t, i}+\sum_{h=1}^{N_{3}} P_{h, i}\right)-\left(P_{\text {Load }, i}+P_{\text {Loss }, i}\right)=0
$$

\section{THE PROPOSED METHOD FOR THE CONSIDERED PROBLEM}

\subsection{Conventional Particle Swarm Optimization (PSO)}

Kennedy and Eberhart [19] first developed PSO in 1995 for reaching optimal variables of benchmark optimization problems. PSO was then improved to be applied for the same optimization problems but better optimal solutions and faster search were required [20-22]. PSO has three different factors including velocity, position and fitness function where fitness function is used to evaluate the effectiveness of position and velocity is used to update new position. The three main factors are formulated as follows [29-30]:

$$
\begin{gathered}
V_{m}^{\text {new }}=V_{m}+c_{1} \cdot \alpha_{1} \cdot\left(\operatorname{Local}_{m}-\operatorname{Pos}_{m}\right)+c_{2} \cdot \alpha_{2} \cdot\left(\operatorname{Pos}_{\text {best }}-\operatorname{Pos}_{m}\right) \\
\operatorname{Pos}_{m}^{\text {new }}=\operatorname{Pos}_{m}+V_{m}^{\text {new }} \\
\text { Fit }_{m}^{\text {new }}=F\left(\operatorname{Pos}_{m}^{\text {new }}\right)
\end{gathered}
$$

Where

$V_{m}^{\text {new }}$ and $V_{m}$ are new and old velocity of the $m$ th individual,

Local $_{m}$ and Pos $_{m}$ are the so-far best and current position of the mth individual,

$\operatorname{Pos}_{m}^{\text {new }}$ is new position of the $m$ th individual,

Pos best is position of the best individual,

$\alpha_{1}$ and $\alpha_{2}$ are random values within 0 and 1 ,

$c_{1}$ and $c_{2}$ are acceleration factors selected within 0 and 2.05,

$\mathrm{Fit}_{m}^{\text {new }}$ is new fitness of the $m t h$ individual.

\subsection{The proposed high-performance PSO (HPPSO)}

The velocity of the PSO above was considered to be ineffective since it did not consider the change during the search process. So, constriction factor and weight factor were suggested to be applied for narrowing the search 
space more effectively [20-21]. Then, velocity update with constriction factor [20] and with inertia weight factor [21] were built as follows:

$$
\begin{aligned}
& V_{m}^{\text {new }}=C F \times\left[V_{m}+c_{1} \cdot \alpha_{1} \cdot\left(\text { Local }_{m}-\operatorname{Pos}_{m}\right)+c_{2} \cdot \alpha_{2} \cdot\left(\operatorname{Pos}_{\text {best }}-\operatorname{Pos}_{m}\right)\right] \\
& V_{m}^{\text {new }}=\left[I W F \cdot V_{m}+c_{1} \cdot \alpha_{1} \cdot\left(\text { Local }_{m}-\operatorname{Pos}_{m}\right)+c_{2} \cdot \alpha_{2} \cdot\left(\operatorname{Pos}_{\text {best }}-\operatorname{Pos}_{m}\right)\right]
\end{aligned}
$$

Where:

$$
\begin{gathered}
C F=\frac{2}{\left|2-\left(\sqrt{\left(c_{1}+c_{2}\right)^{2}-4\left(c_{1}+c_{2}\right)}+\left(c_{1}+c_{2}\right)\right)\right|} \\
I W F=\frac{I W F_{\max }-I W F_{\min }}{\text { NIter }_{\max }} I W F_{\max } \text { NIter }
\end{gathered}
$$

Where

$I W F$ and $C F$ are inertia weight factor and constriction factor.

$I W F_{\max }$ and $I W F_{\min }$ are the highest and lowest values of inertia weight factor NIter $_{\text {max }}$ and Niter are the highest and the current iteration

In addition, PSO was also suggested to be modified by improving acceleration coefficients [17]. The two coefficients were varied from the lowest to the highest value similarly to inertia weight factor. For this case, the velocity is updated by:

$$
V_{m}^{\text {new }}=\left[V_{m}+c_{1}^{\prime} \cdot \alpha_{1} \cdot\left(\operatorname{Local}_{m}-\operatorname{Pos}_{m}\right)+c_{2}^{\prime} \cdot \alpha_{2} \cdot\left(\operatorname{Pos}_{\text {best }}-\operatorname{Pos}_{m}\right)\right]
$$

where

$$
\begin{gathered}
c_{1}^{\prime}=c_{1, \text { start }}+\left(c_{1, \text { end }}-c_{1, \text { start }}\right) \frac{\text { NIter }}{\text { NIter }_{\max }} \\
c_{2}^{\prime}=c_{2, \text { start }}+\left(c_{2, \text { end }}-c_{2, \text { start }}\right) \frac{\text { NIter }}{\text { NIter }_{\max }}
\end{gathered}
$$

Where

$c_{1}^{\prime}$ and $c_{2}^{\prime}$ are modified version of $c_{1}$ and $c_{2}$,

$c_{1, \text { start }}$ and $c_{2, \text { start }}$ are initial values of of $c_{1}^{\prime}$ and $c_{2}^{\prime}$,

$c_{1, \text { end }}$ and $c_{2, \text { end }}$ are final values of $c_{1}^{\prime}$ and $c_{2}^{\prime}$.

The PSO with two time-varying acceleration factors obtained by (19) and (20) is called TVAC-PSO. On the other hand, PSO with the use of both IWF and CF obtained by using (14) and (15) is called FCIW-PSO. In this paper, we suggest combining constriction factor, inertia weight factor and modified acceleration coefficients for updating new velocity. As a result, the new velocity is formulated by:

$$
V_{m}^{\text {new }}=C F\left[I W F \cdot V_{m}+c_{1}^{\prime} \cdot \alpha_{1} \cdot\left(\operatorname{Local}_{m}-\operatorname{Pos}_{m}\right)+c_{2}^{\prime} \cdot \alpha_{2} \cdot\left(\operatorname{Pos}_{\text {best }}-\operatorname{Pos}_{m}\right)\right]
$$

\subsection{The application of HPPSO for the problem}

The whole search process of HPPSO for the problem is shown in Figure 2 and described as follows:

Step 1: Set value to population and the maximum iteration

Step 2: Randomly produce $\operatorname{Vol}_{h, i}$ and $P_{t, i}\left(\right.$ for $\left.i=2, \ldots, N_{3}\right)$ for each solution $\operatorname{Pos}_{m}$

Step 3: Calculate $\operatorname{Dis}_{h, I}, P_{h, i}$ and $P_{t, i}$ (for $t=1$ )

Step 4: Calculate fitness function and set NIter to 1

Step 5: Calculate new velocity and new position using (21) and (12)

Step 6: Check boundaries and correct if violation happen

Step 7: Calculate $D i s_{h, I}, P_{h, i}$ and $P_{t, i}($ for $t=1)$

Step 8: Calculate fitness function and compare to keep better solution

Step 9: Determine Pos best and Local $_{m}$

Step 10: If NIter $=$ NIter $_{\max }$, stop search algorithm. Otherwise, assign NIter $=$ NIter+1 and back to Step 5.

IJEEI, Vol.8, No. 4, December 2020: 648 - 657 


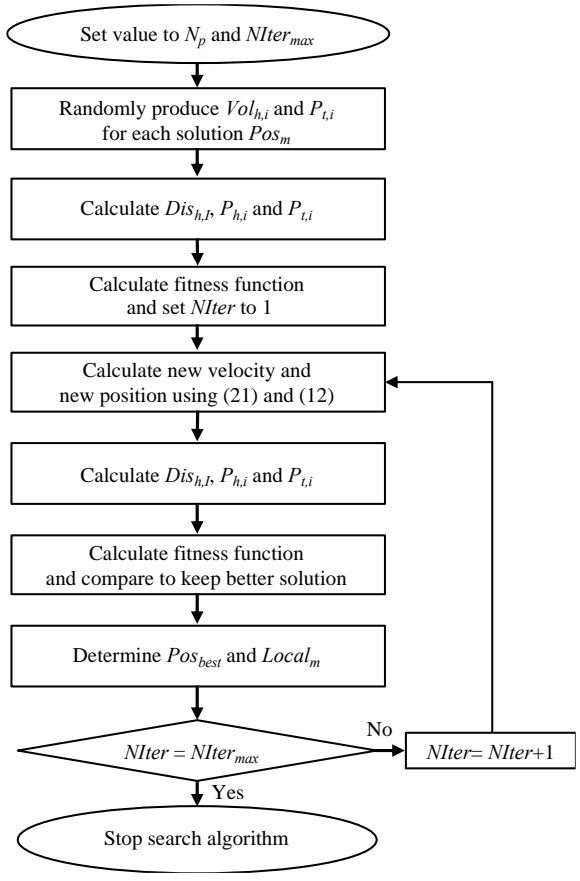

Figure 2. Main steps of implementing HPPSO for the considered problem

\section{NUMERICAL RESULTS}

In this section, the proposed HPPSO is evaluated by comparing results of the method to those from other previous ones and other implemented methods such as PSO, FCIW-PSO, TVAC-PSO, SSA and HHA. A test system with one HdP and one ThP is optimally scheduled over six twelve-hour subperiods [6]. The six methods are coded on Matlab and personal computer with CPU of Intel Core i7-2.4GHz-RAM 4GB for reaching 100 successful runs.

\subsection{Results from the implemented methods}

Results in details obtained by six methods are reported in Table 1 . All the implemented methods are run

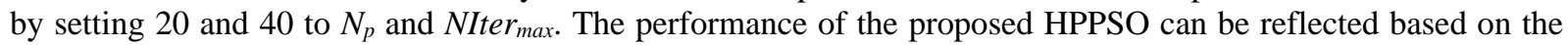
comparison criteria below:

1) The minimum EPGC: This value indicates the strong search ability of methods. Lower minimum EPGC means better solution found and method with lower EPGC is much stronger.

2) The mean EPGC: This is the average value of 100 solutions. So, lower average value means much more stability and method with lower average is more stable than other ones. In addition, standard deviation and the fitness function of all successful runs are also reflected the same manner.

3) The maximum EPGC: This is the maximum EPGC over 100 solutions. So, higher value means worst solution is found.

4) The best convergence, mean and worst convergence characteristic: The curves indicate the search speed of compared methods.

Table 1. Comparison of results obtained by implemented methods

\begin{tabular}{cccccc}
\hline \multirow{2}{*}{ Method } & $\begin{array}{c}\text { Minimum EPGC } \\
(\$)\end{array}$ & $\begin{array}{c}\text { Average EPGC } \\
(\$)\end{array}$ & $\begin{array}{c}\text { Maximum EPGC } \\
(\$)\end{array}$ & $\begin{array}{c}\text { Standard deviation } \\
(\$)\end{array}$ & $\begin{array}{c}\text { Computation time } \\
(\mathrm{s})\end{array}$ \\
\hline PSO & 710241.23 & 722207.46 & 736951.66 & 9762.62 & 0.03 \\
TVAC-PSO & 709864.96 & 710130.33 & 712719.1 & 561.35 & 0.03 \\
FCIW-PSO & 709865.36 & 710664.94 & 720439.18 & 1450.45 & 0.03 \\
SSA & 709878.47 & 711170.53 & 727938.81 & 2141.3291 & 0.03 \\
HHA & 710808.19 & 719958.19 & 734081.22 & 4528.76 & 0.03 \\
HPPSO & 709862.0489 & 709900.936 & 711811.468 & 224.504 & 0.03 \\
\hline
\end{tabular}

So, the best characteristic and the mean characteristic are also plotted for comparison in addition to numerical results in Table 1. Table 1 indicates that HPPSO can reach lower minimum EPGC, lower mean EPGC, 
lower maximum EPGC and lower standard deviation than other ones. As running the six methods, \$709862.0489 is the lowest EPGC of the system and there is no better-found solution with lower EPGC than this value. The proposed HPPSO can find 33 solutions with the same EPGC as \$709862.0489 whereas other ones cannot find even one solution with the EPGC. Figure 3, Figure 4 and Figure 5 show much faster speed of HPPSO as compared to other ones for the three convergence characteristics. The solution of HPPSO at the $20^{\text {th }}$ iteration is much faster than that of others at the final iteration. Furthermore, fitness function of 100 runs from HPPSO and other methods shown in Figure 6 is also a good evidence of outstanding robustness of HPPSO over others. 100 values of EPGC from HPPSO in red are approximately equal excluding a few values. These values of others are much higher than those of HPPSO and they have very high fluctuations.

Derived from the analysis above, it can conclude as follows:

1) HPPSO can find more optimal operation parameters than other applied approaches

2) HPPSO is always convergent to more optimal operation parameters

3) HPPSO is much quicker than others

The optimal solutions found by these applied methods in Table 1 are reported in Table 2.

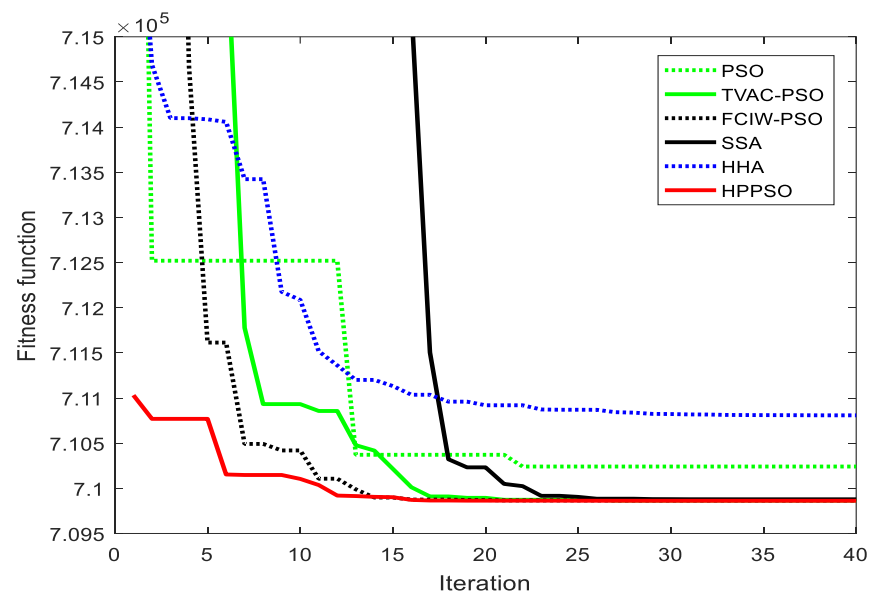

Figure 3 . The best convergence obtained by the six implemented methods

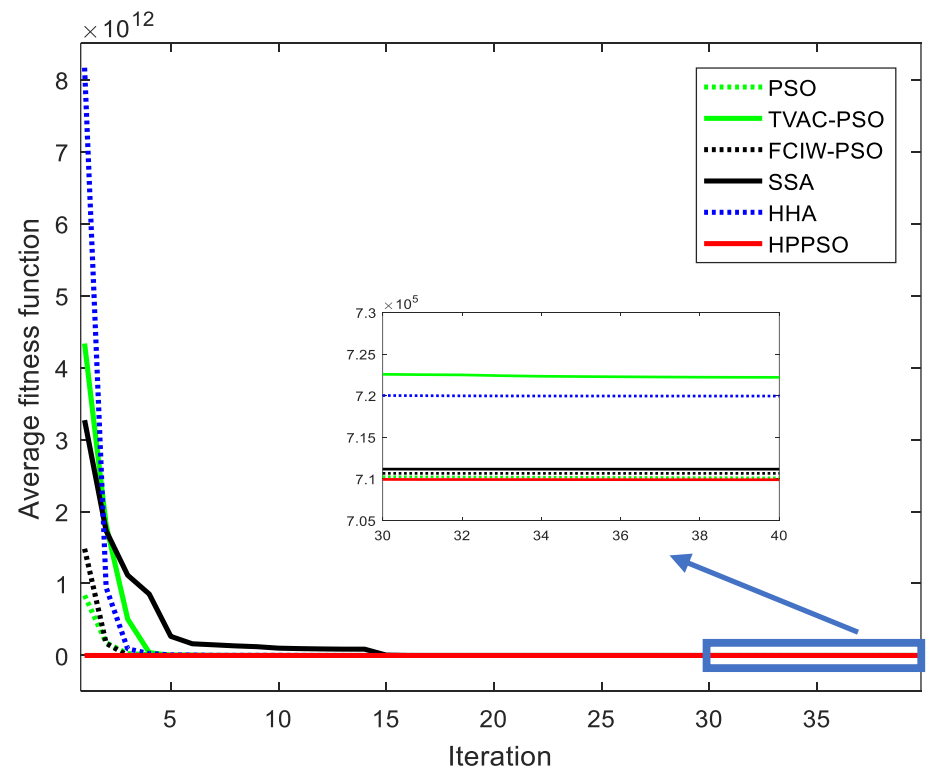

Figure 4. The mean convergence of 100 successful runs

IJEEI, Vol.8, No. 4, December 2020: 648 - 657 


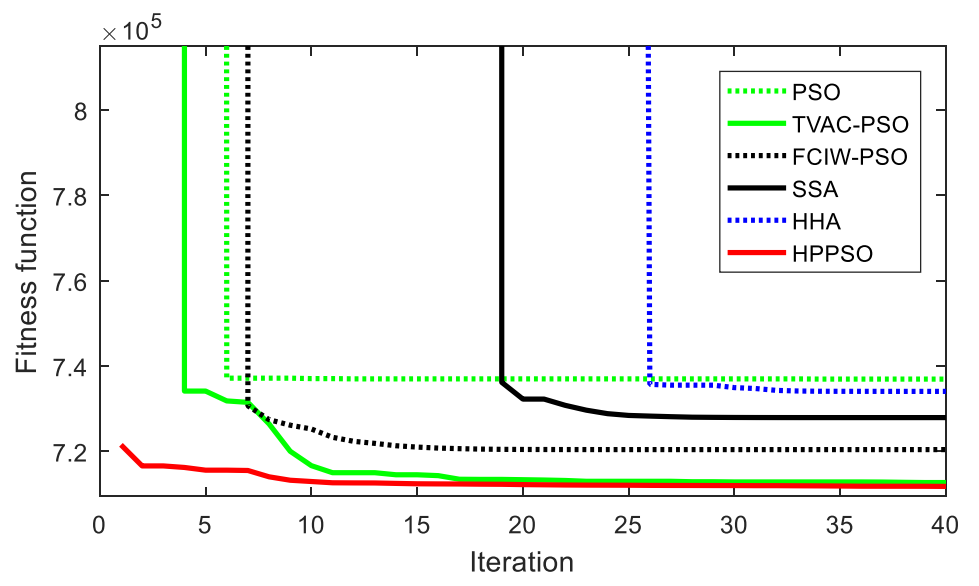

Figure 5. The worst convergence obtained by the six implemented methods

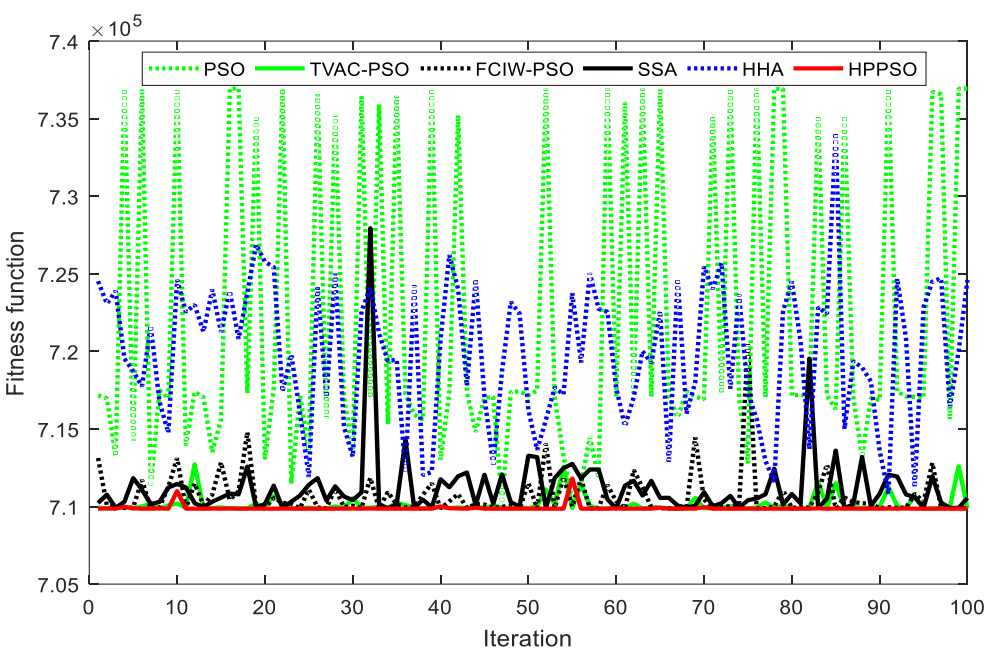

Figure 6. Fitness function of 100 runs obtained by six implemented methods

Table 2. Optimal control variable values found by applied methods

\begin{tabular}{ccccccc}
\hline$i$ & PSO & TVAC-PSO & FCIW-PSO & SSA & HHA & HPPSO \\
\hline 1 & 104968.078 & 102102.161 & 101786.129 & 100949.606 & 101807.695 & 101807.695 \\
2 & 88033.081 & 86472.556 & 86344.208 & 84764.958 & 83151.825 & 83151.825 \\
3 & 96670.317 & 94366.866 & 94217.578 & 93865.750 & 88506.936 & 88506.936 \\
4 & 60415.019 & 60005.517 & 60000.008 & 60000.009 & 62806.247 & 62806.247 \\
5 & 75070.935 & 70434.314 & 70671.718 & 70735.533 & 67818.421 & 67818.421 \\
6 & 60000.000 & 60000.000 & 60000.000 & 60000.000 & 60000.000 & 60000.000 \\
\hline
\end{tabular}

\subsection{Comparisons with previous approaches}

In this part, HPPSO is also compared with other ones in previous studies shown in Table 3 . The best EPGC indicates that HPPSO can find either the same solutions or better solutions than other ones, especially much better solutions than GSA, SSA, GA and EPA. Although other methods can find the same EPGC as HPPSO, these methods have been run by setting much higher population and more iterations. The overview through these values shows that population of others is from 8 to 60 and the maximum iteration is from 70 to 500 but only 20 and 40 are set for HPPSO. In addition, it has taken HPPSO 0.03 second but others from 4.54 to 2640 seconds. Clearly, HPPSO is much more favorable than previous approaches. 
Table 3. Comparison of the best EPGC by the proposed method and previous methods

\begin{tabular}{cccccccccc}
\hline Method & Best EPGC $(\$)$ & $N p$ & Iter $_{\max }$ & Cpu $(\mathrm{s})$ & Method & Best EPGC $(\$)$ & $N p$ & Iter $\max$ & Cpu (s) \\
\hline GSA [2] & 709877.38 & - & - & - & CSOA [13] & 709862.05 & 30 & 70 & 4.54 \\
SAA [9] & 709874.36 & - & 200 & 901 & LORCSA [14] & 709862.049 & 10 & 300 & 0.18 \\
GA [10] & 709863.56 & 30 & 300 & - & CORCSA [14] & 709862.049 & 10 & 300 & 0.18 \\
EPA [10] & 709862.06 & 30 & 300 & 8 & LCSA [15] & 709862.049 & 30 & 400 & 0.3 \\
EPA [11] & 709863.29 & 50 & 400 & 2640 & CCSA [15] & 709862.049 & 30 & 400 & 0.3 \\
MEPA [12] & 709862.05 & 60 & 500 & 159.18 & GCSA [15] & 709862.049 & 30 & 400 & 0.3 \\
FEPA [12] & 709862.05 & 60 & 300 & 101.4 & ACSA [16] & 709862.050 & 8 & 100 & 0.12 \\
IFEPA [12] & 709862.05 & 60 & 150 & 59.7 & ASCSA [19] & 709862.049 & 20 & 40 & 0.03 \\
RIFEPA [12] & 709862.05 & - & 300 & - & HPPSO & 709862.049 & 20 & 40 & 0.03 \\
\hline
\end{tabular}

\section{CONCLUSION}

In this paper, six methods including PSO, TVAC-PSO, FCIW-PSO, SSA, HHA and HPPSO have been applied for solving the STHTO problem. Six implemented methods have been run by setting the same values to population size and iterations but obtained results were totally different. The HPPSO could reach the global solutions many times over 100 successful runs but other ones have failed to find global solutions even for one time. The minimum EPGC, average EPGC, maximum EPGC and standard deviation are necessary evidences for demonstrating a real outstanding performance of HPPSO over five other ones. In addition, convergence characteristics also indicated that HPPSO was at least two times faster than other implemented ones. Similarly, the comparisons with previous methods have shown the same evaluation since HPPSO could reach the same or better solutions than other ones; however, HPPSO has used much lower values for control parameters and spent much shorter computation time. So, it can conclude that HPPSO should be used for the systems with ThPs and HdPs. And in future work, the method will be applied for the larger system dimensions with wind turbines, photovoltaic and hydrothermal systems.

\section{REFERENCES}

[1] Shaaban, M., Zeynal, H., \& Nor, K. "MILP-based short-term thermal unit commitment and hydrothermal scheduling including cascaded reservoirs and fuel constraints," International Journal of Electrical \& Computer Engineering (20888708), vol. 9, 2019.

[2] Wood, A. J., Wollenberg, B. F., \& Sheblé, G. B. "Power generation, operation, and control,” John Wiley \& Sons.

[3] Arya, Y. "A new optimized fuzzy FOPI-FOPD controller for automatic generation control of electric power systems," Journal of the Franklin Institute, Vol. 356(11), 5611-5629, 2019.

[4] Arya, Y. "Effect of energy storage systems on automatic generation control of interconnected traditional and restructured energy systems," International Journal of Energy Research, Vol. 43(12), 6475-6493, 2019.

[5] Gupta, S. K., Arya, Y., Shukla, S., \& Chawla, P. "Two-area AGC in interconnected system under the restructured power system using BFO controller,” In 2014 6th IEEE Power India International Conference (PIICON) (pp. 1-6). IEEE, 2014, December.

[6] Dahiya, P., Mukhija, P., Saxena, A. R., \& Arya, Y. "Comparative performance investigation of optimal controller for AGC of electric power generating systems," Automatika: časopis za automatiku, mjerenje, elektroniku, računarstvo $i$ komunikacije, 57(4), 902-921, 2016.

[7] Arya, Y. "AGC of PV-thermal and hydro-thermal power systems using CES and a new multi-stage FPIDF-(1+ PI) controller," Renewable energy, 134, 796-806, 2019.

[8] Zaghlool, M. F., Trutt, F. C. "Efficient methods for optimal scheduling of fixed head hydrothermal power systems," IEEE transactions on power systems, vol. 3, pp. 24-3, 1988.

[9] Wong, K. P., Wong, Y. W. "Short-term hydrothermal scheduling part. I. Simulated annealing approach," IEE Proceedings-Generation, Transmission and Distribution, vol. 141, pp. 497-501, 1994.

[10] Yang, P. C., Yang, H. T., Huang, C. L. "Scheduling short-term hydrothermal generation using evolutionary programming techniques," IEE Proceedings-Generation, Transmission and Distribution, vol. 143, pp. 371-376, 1996.

[11] Hota, P. K., Chakrabarti, R., Chattopadhyay, P. K. "Short-term hydrothermal scheduling through evolutionary programming technique,” Electric Power Systems Research, vol. 52, pp. 189-196, 1999.

IJEEI, Vol.8, No. 4, December 2020: 648 - 657 
[12] Sinha, N., Chakrabarti, R., Chattopadhyay, P. K. "Fast evolutionary programming techniques for short-term hydrothermal scheduling," IEEE transactions on Power Systems, vol. 18, pp. 214-220, 2003.

[13] Türkay, B., Mecitoğlu, F., Baran, S. "Application of a fast evolutionary algorithm to short-term hydro-thermal generation scheduling," Energy Sources, Part B: Economics, Planning, and Policy, vol. 6, pp. 395-405, 2011.

[14] Swain, R. K., Barisal, A. K., Hota, P. K., Chakrabarti, R. "Short-term hydrothermal scheduling using clonal selection algorithm," International Journal of Electrical Power \& Energy Systems, vol. 33, pp. 647-656, 2011.

[15] Nguyen, T. T., Vo, D. N., Dinh, B. H. "Cuckoo search algorithm using different distributions for short-term hydrothermal scheduling with reservoir volume constraint," International Journal on Electrical Engineering and Informatics, vol. 8, 76, 2016.

[16] Nguyen, T. T., Vo, D. N., Ongsakul, W. "One rank cuckoo search algorithm for short-term hydrothermal scheduling with reservoir constraint," In 2015 IEEE Eindhoven PowerTech, IEEE, pp. 1-6, 2015.

[17] Dinh, B. H., Nguyen, T. T., Vo, D. N. "Adaptive cuckoo search algorithm for short-term fixed-head hydrothermal scheduling problem with reservoir volume constraints," International Journal of Grid and Distributed Computing, vol. 9 , pp.191-204, 2016.

[18] Nguyen, T. T., Vo, D. N., Deveikis, T., Rozanskiene, A. "Improved cuckoo search algorithm for nonconvex hydrothermal scheduling with volume constraint," Elektronika ir Elektrotechnika, vol. 23, 68-73, 2017.

[19] Nguyen, T. T., Vo, D. N., Dinh, B. H. "An effectively adaptive selective cuckoo search algorithm for solving three complicated short-term hydrothermal scheduling problems," Energy, vol. 155, pp. 930-956, 2018.

[20] Kennedy, J., Eberhart, R. "Particle swarm optimization," In Proceedings of the ICNN'95-International Conference on Neural Networks, Perth, Australia, pp. 1942-1948, 1995.

[21] Clerc, M. "The swarm and the queen: Towards a deterministic and adaptive particle swarm optimization," In Proceedings of the 1999 Congress on Evolutionary Computation-CEC99 (Cat. No. 99TH8406), Washington, DC, USA, vol. 6-9, pp. 1951-1957, 1999.

[22] Eberhart, R.C., Shi, Y.H. "Comparing inertia weights and constriction factors in particle swarm optimization," In Proceedings of the IEEE Congress on Evolutionary Computation, La Jolla, CA, USA, 16-19; pp. 84-88, 2000.

[23] Ratnaweera, A., Halgamuge, S.K., Watson, H.C. "Self-organizing hierarchical particle swarm optimizer with time-varying acceleration coefficients," IEEE Trans. Evol. Comput., vol.8, pp. 240-255, 2004.

[24] Mirjalili, S., Gandomi, A.H., Mirjalili, S.Z., Saremi, S., Faris, H., Mirjalili, S.M. "Salp Swarm Algorithm: A bio-inspired optimizer for engineering design problems," Adv. Eng. Softw, vol. 114, pp. 163-191, 2017.

[25] Heidari, A. A., Mirjalili, S., Faris, H., Aljarah, I., Mafarja, M., \& Chen, H. "Harris hawks optimization: Algorithm and applications," Future generation computer systems, vol. 97, pp. 849-872, 2019.

[26] Ajenikoko, G. A., Olakunle, O., \& Olabode, E. (2017). Optimal Power Flow with Reactive Power Compensation for Cost and Loss Minimization on Nigerian Power Grid System. Indonesian Journal of Electrical Engineering and Informatics (IJEEI), 5(3), 236-247.

[27] Rajalakshmi P, Rathinakumar M. "An optimal voltage stability enhancement by locating FACTS in optimal place," Indonesian Journal of Electrical Engineering and Informatics (IJEEI), vol. 7, no. 1, pp. 105 116, 2019.

[28] Lenin, K. "Minimization of real power loss by enhanced teaching learning based optimization algorithm. IAES International Journal of Robotics and Automation, vol 9 no. 1, pp. 1-5, 2020.

[29] Hasan, A. M. "A hybrid approach of using particle swarm optimization and volumetric active contour without edge for segmenting brain tumors in MRI scan," Indonesian Journal of Electrical Engineering and Informatics (IJEEI), vol. 6 no. 3, 292-300, 2018.

[30] Jaikhang, W., \& Tunyasrirut, S. "Artificial Intelligent Tuning PI Controller on Wind Turbine System with Three-Phase Grid Connected System." Indonesian Journal of Electrical Engineering and Informatics (IJEEI), vol. 7 no. 4, pp. 703$719,2019$. 